\title{
INTEGRATION OF THE RESPONSE SURFACE METHODOLOGY WITH THE COMPROMISE DECISION SUPPORT PROBLEM IN DEVELOPING A GENERAL ROBUST DESIGN PROCEDURE
}

\author{
Wei Chen \\ Systems Realization Laboratory, G.W. Woodruff School of Mechanical Engineering
}

Kwok-Leung Tsui

School of Industrial and Systems Engineering

Janet K. Allen and Farrokh Mistree

Systems Realization Laboratory, G.W. Woodruff School of Mechanical Engineering

Georgia Institute of Technology

Atlanta, Georgia

\section{ABSTRACT}

In this paper we introduce a comprehensive and rigorous robust design procedure to overcome some limitations of the current approaches. A comprehensive approach is general enough to model the two major types of robust design applications, namely,

- robust design associated with the minimization of the deviation of performance caused by the deviation of noise factors (uncontrollable parameters), AND

- robust design due to the minimization of the deviation of performance caused by the deviation of control factors (design variables).

We achieve mathematical rigor by using, as a foundation, principles from the design of experiments and optimization. Specifically, we integrate the Response Surface Method (RSM) with the compromise Decision Support Problem (DSP). Our approach is especially useful for design problems where there are no closed-form solutions and system performance is computationally expensive to evaluate. The design of a solar powered irrigation system is used as an example. Our focus in this paper is on illustrating our approach rather than on the results per se.

\section{OUR FRAME OF REFERENCE}

The fundamental principle in robust design is to improve the quality of a product by minimizing the effects of variation without eliminating these causes. There are two broad categories, of robust design based on the source of variation:

Type I Robust Design - minimizing performance deviation caused by deviation of noise factors (uncontrollable parameters).

Type II Robust Design - minimizing performance deviation caused by control factor deviation (design variables).
The Taguchi method was developed for Type I applications (Phadke, 1989). This is basically a two-part orthogonal array for experimental design using the signal-to-noise-ratio as an optimization criterion. Although applications of the Taguchi method have yielded useful results, this method has also been criticized by statisticians (Box, 1988, Nair, 1992, Palatino, Tsui, 1992). Tsui, 1992, argues that many of Taguchi's statistical methods, e.g., orthogonal arrays, linear graphs and accumulation analysis, are not statistically efficient and proposes to overcome these limitations using alternative experimental formats, design criteria, analysis techniques, graphical tools and optimization strategies. Box, 1988, points out that there are various mathematical difficulties/ requirements associated with the use of signal-to-noise-ratio. Alternatively, Welch, et al., 1990, propose combining control and noise factors into a single array thus modeling the response rather than expected loss, and approximating a prediction model for loss based on the fittedresponse model, this approach is further developed by Shoemaker, et al., 1991. The proposed modifications to the Taguchi method, however, involve a single performance metric. In our opinion, as there are multiple objectives to be satisfied in design it follows that there must be multiple aspects to quality. This opinion is reinforced by some preliminary insight we gained using quality engineering techniques to reduce the number of the trajectory simulations of a LifeSat space vehicle (Mistree, et al., 1993b). Further, we investigated the benefits and limitations of using the Taguchi method in the early stages of design by applying it to concurrent concept selection and system synthesis of a solar powered irrigation system (Chen, et al., 1994). We assert that quality loss depends on several quality characteristics and each of these may have different degrees of importance. Related to this view, Otto and Antonsson, 1991, argue the necessity of incorporating constraints in robust design. 
On another front, nonlinear programming methods can be used for both Type I and II applications. Ramakrishnana and Rao, 1991, formulate the robust design problem as a nonlinear optimization problem with Taguchi's loss function as the objective. They emphasize the numerical evaluation of total quality loss but fall short in addressing the nature of variations. Sundaresan et al., 1993, incorporate a Sensitivity Index (SI) in the optimization procedure to determine a "robust optimum". The SI is defined as the root mean square value of the difference between the value of the function for the worst case and the target value. In this case, it is difficult to determine the weighting factors for target performance and variance in the objective function as designers may be uncertain about the ranges for the actual performance and its variance. When using nonlinear programming methods, Sundaresan et al. use concepts from the design of experiments to approximate the variation in performance at each iteration. However, this is computationally inefficient for large, analysis-intensive and computationallyintensive problems.

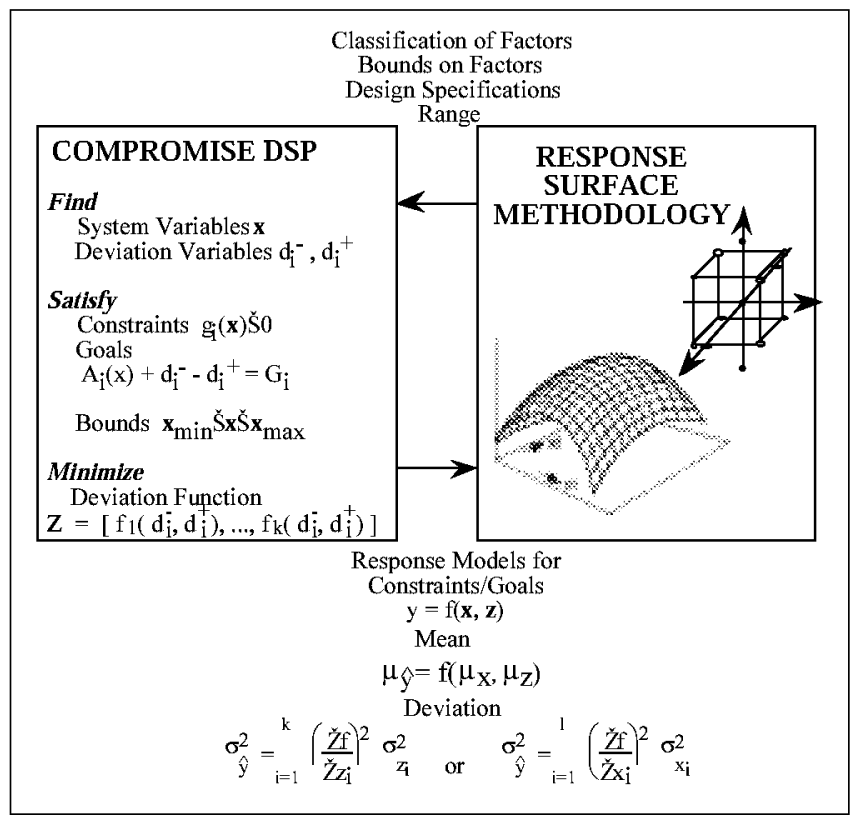

\section{FIGURE 1. INTEGRATION OF RESPONSE SURFACE METHODOLOGY WITH THE COMPROMISE DSP}

Our focus in this paper is to introduce a comprehensive and rigorous robust design procedure to overcome some of these limitations. By comprehensive, we mean that our approach is general enough to model the two major types of robust design applications, namely, robust design to minimize the deviation of noise factors (uncontrollable parameters), AND robust design to minimize deviation of control factors (design variables). Thus, our approach is general enough to be used for both Type I and II robust design. We achieve mathematical rigor by using principles from the design of experiments and optimization. Specifically, we integrate the Response Surface Method (RSM) with the compromise Decision Support Problem (DSP), Figure 1. The
Response Surface Method (RSM) is a collection of statistical techniques which support the design of experiments and fitting a response model (Khuri and Cornell, 1987). The compromise DSP is a multiobjective decision model (Mistree, et al., 1993a) which enables a designer to determine values of design variables which satisfy a set of constraints to achieve a set of goals. The objective is to minimize the deviations of different goals from target values using lexicographic minimization (Ignizio, 1985).

We build approximating functions using the response surface model to relate each response to all important control- and noisefactors. Then we solve the problem using the compromise DSP and these approximations rather than solving the problem directly using a computationally expensive analysis model. In this way, the variance of performance can be predicted instead of running multiple experiments at each iteration of the solution. The compromise DSP provides a generic approach to achieve robust design by enabling a designer to find values of control factors to achieve a performance which is as close as possible to the targets and to minimize variations around the targets, subject to engineering constraints. Using the compromise DSP, it is possible to address individually the issues of maximizing the intensity of the signal on target and minimizing variation. These become separate goals in the multiobjective compromise DSP. This approach helps a designer focus on individual contributions to mean and variation and to identify parameters which affect the attainment of specific goals.

In the following, we discuss the two major types of robust design. We then show the integration of Response Surface Method and the compromise DSP into a general procedure for robust design. The design of a solar powered irrigation system is used as an example. Our focus in this paper is on explaining the approach rather than on the results per se.

\section{A DESCRIPTION OF OUR APPROACH}

\section{Two Types of Robust Design}

The concepts behind the two major types of robust design application are illustrated in Figure 2. On the left-hand side of Figure 2, we use a P-diagram (Phadke, 1987) to represent different types of parameters in robust design, their relationships with the whole system, and thus the differences in source of variation in response for Type I and Type II applications. Control factors $(\boldsymbol{x})$ are parameters which can be specified freely by a designer; noise factors (z) are parameters that are not under a designer's control; and the signal factor $(\boldsymbol{M})$ is the intended value for the response $(y)$ of a product/process. In Type I applications, the variance of the response is caused by variations in the noise factor, z. Type II is different from Type I in that its input does not include a noise factor. The variation in performance is caused solely by variations in control factors or design variables in the region $\pm \Delta x$.

On the right hand side of Figure 2 we present a schematic of the different concepts behind the two types robust design. Taguchi's robust design method is a Type I method and is highlighted in the upper right block of Figure 2. In the Taguchi method, a designer adjusts control factors, $\boldsymbol{x}$, to dampen the variations caused by the noise factor, $z$. The two curves represent 
the performance variation as a function of noise factor when $\boldsymbol{x}$ is at two different levels, $\boldsymbol{x}=\mathrm{a}$ and $\boldsymbol{x}=\mathrm{b}$. If the design objective is to achieve a performance as close as possible to the target, $M$, the designs at both levels are acceptable because their means are the target $\boldsymbol{M}$. However, introducing robustness, when $\boldsymbol{x}=\mathrm{a}$, the performance varies significantly with the deviation of noise factor, $\boldsymbol{z}$, however when $\boldsymbol{x}=\mathrm{b}$, the performance deviates much less. Therefore, $\boldsymbol{x}=\mathrm{b}$ is a better than $\boldsymbol{x}=\mathrm{a}$ as a design solution which dampens the effect of the noise factors.
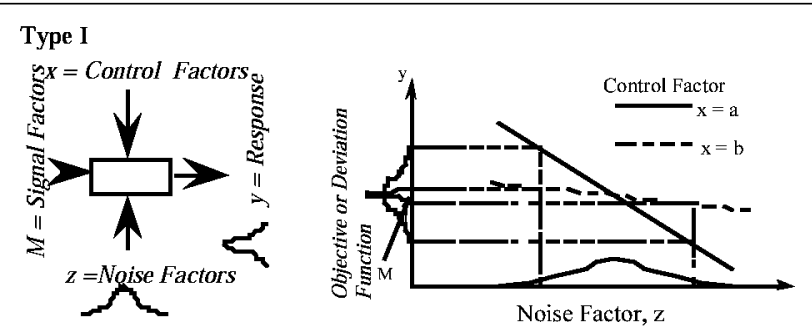

Type II
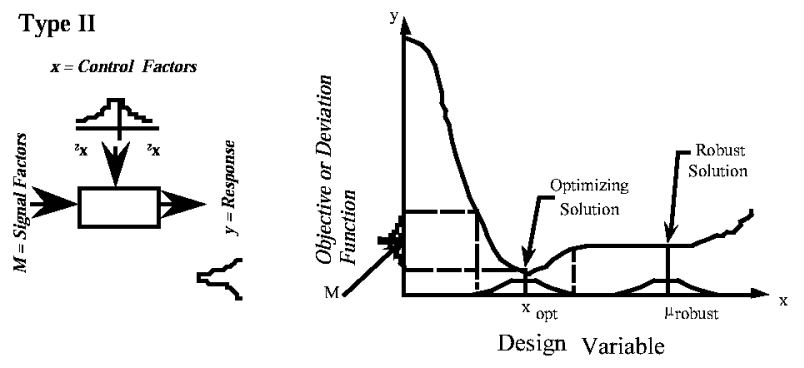

FIGURE 2. A COMPARISON OF TWO TYPES
OF ROBUST DESIGN

The concept behind Type II robust design is represented in the lower right block of Figure 2. For purposes of illustration, assume that performance is a function of only one variable, $\boldsymbol{x}$. Generally, in this type of robust design, to reduce the variation of response caused by the deviations of design variables, instead of seeking the optimum value, a designer is interested in the flat part of a curve near the performance target. If the objective is to move the performance function towards target $M$ and if a robust design is not sought then obviously the point $\mathrm{x}=\mathrm{a}$ is chosen. However, for a robust design, $x=b$ is a better choice. This is because if design variables vary within the region $\pm \Delta x$ of their means, the resulting variation of response of the design at $x=b$ is much smaller than that at $\mathrm{x}=\mathrm{a}$, while the means of the response at two designs are close.

Although the concepts behind the two major types of robust design are different, robust design is always concerned with aligning the peak of the bell shaped response distribution with the targeted quality, (bring the mean to the target) and making the bell shaped curve thinner (reduce the variance). This makes it possible to develop a general procedure for robust design in which these objectives are modeled as separate goals in a compromise DSP.

\section{Developing a General Robust Design Procedure}

As mentioned in Section I, to overcome the limitations of Taguchi's loss model approach, we use the Response Surface Method, RSM, starting with the response-model approach (Welch, et al., 1990: Shoemaker, et al., 1991). The major elements of the response-model approach are:

- Combining control and noise factors in a single array instead of using Taguchi's inner- and outer-array approach,

- modeling the response itself rather than expected loss,

- approximating a prediction model for loss based on the fitted-response model.

The RSM, is used to support the design of experiments and fitting a response model. Instead of using Taguchi's orthogonal array as the combined array for experiments, other fractional factorial designs or central composite designs can be chosen. In general, the response-model postulates a single, formal model of the type

$$
\hat{y}=f(x, z),
$$

where $\hat{y}$ is the estimated response scalar and $\mathbf{x}$ and $\mathbf{z}$ are the settings of the control and noise variables. The surface model is usually modeled with a second order equation, from this, it is possible to estimate response mean and variability. For Type I applications in which the deviations of noise factors are the sources of variation:

Mean of the response $\quad \mu_{\mathrm{y}}=\mathrm{f}\left(\mathrm{X}, \mu_{\mathrm{Z}}\right)$

Variance of the response $\sigma_{\hat{y}}^{2}={ }_{i=1}^{k}\left(\frac{\partial f}{\partial z_{i}}\right)^{2} \sigma_{z i}^{2}$

where $\mu$ is the mean value, $\mathrm{k}$ is the number of noise factors in the response model and $\sigma_{\mathrm{Zi}}$ is the standard deviation of each noise factor. In Eqn. (1.3), it is assumed that the noise variables are independent. In Type II robust design, i.e., when the deviations of control factors are the source of variation, Eqns. (1.2) and (1.3) are modified:

$$
\begin{array}{ll}
\text { Mean of the response } & \mu_{\mathrm{xy}}=\mathrm{f}(\mathrm{x}) \\
\text { Variance of the response } & \sigma_{\hat{\mathrm{y}}}^{2}={ }_{\mathrm{i}=1}^{1}\left(\frac{\partial \mathrm{f}}{\partial \mathrm{x}_{\mathrm{i}}}\right)^{2}{ }^{2} \sigma_{\mathrm{x}_{\mathrm{i}}}^{2} .
\end{array}
$$

Using the response-model approach, robust design for either type of application can be achieved by bringing the mean given in Eqn (1.2) or (1.4) to the target and minimizing the variance, Eqn. (1.3) or (1.5). Because the classical RSM is restricted to unconstrained searching for a local optimum of a single response (or the variance of that response) a compromise Decision Support Problem, is introduced to handle multiple quality aspects and engineering constraints.

A typical structure for a compromise DSP is presented in Figure 1 and described in detail elsewhere (e.g., Mistree, et al., 1993a). The compromise DSP assists the designer in modeling and handling multiple trade-offs simultaneously. In the compromise DSP, each goal, $A_{\mathbf{i}}$, has two associated deviation variables $\mathrm{d}_{\mathbf{i}}{ }^{-}$and $\mathrm{d}_{\mathbf{i}}{ }^{+}$which indicate the extent of the deviation from the target $\mathrm{G}_{\mathbf{j}}$. To effect a solution, on the basis of preference, goals may be rank-ordered into priority levels using the lexicographic minimum. (Ignizio 1985). 
In Figure 1, the integration of the compromise DSP and RSM are illustrated. When formulating the compromise DSP, different parameters are classified as system variables, system parameters (constant variables) or system performances (constraints/goals). All the decisions in classifying parameters are used to help building response surface models. Using the quality engineering terminology, response surface models are developed for responses (system performances) as functions of control factors (system variables) and noise factors (system parameters with deviation) over the region of interest (defined by the bounds of design variables). Based on a response surface model, the mean and variance of a response can be derived as functions of control factors according to the sources of variation (either the variation of noise factors or control factors themselves). The explicit forms of these equations are passed to the compromise DSP where they are either used as constraints or goals. Instead of using a single criterion and minimizing the signal-to-noise ratio, an important aspect of this work is to permit a designer to independently bring the mean on target and minimize the variation around this target. This is accomplished by modeling these objectives separately.

We believe this is a comprehensive robust design procedure. If a surface model is established over the space formed by all the parameters involved, the same response surface model can be used for either type of compromise DSP. It is not difficult to imagine that these two formulations can be combined to achieve a robust design where there are deviations of both control and noise factors.

We use a worst case scenario instead of expected values of system performance for constraints to study variations of constraints caused by the deviations of controllable or uncontrollable parameters. Using the expected values of system performance for constraints might permit the constraints to be violated, especially when the constraints are close to being active at the solution point. Using the worst case for the constraints, instead of using $E\left[g_{j}(x, z)\right] \leq 0$,

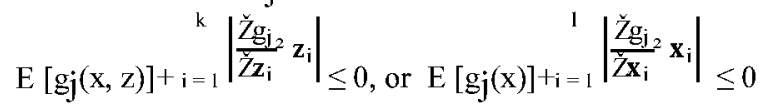

where $\Delta z_{i}$ is the deviation of a noise parameter and $\Delta x_{i}$, the deviation of a sensitive design variable (control factor); $\mathrm{k}$ and 1 are the numbers of noise parameters and sensitive design variables, respectively. Next, we use the design of a solarpowered irrigation system to demonstrate our approach.

\section{A SOLAR POWERED IRRIGATION SYSTEM DESIGN EXAMPLE}

Previously, we have used the solar powered irrigation system design as an example to show the application of Taguchi techniques to concurrent subsystem embodiment and system synthesis (Chen, et al., 1994). We used the inner- and outerarrays for experiments and the "signal-to-noise ratio" as our measurement and found that there are many limitations of the Taguchi's approach, Section 1. It was difficult to use this method when making tradeoffs among multiple quality characteristics and incorporating constraints and factor interactions. In this paper, using the same problem, we show how our newly proposed method can be used to overcome these difficulties and extend the robust design applications. The layout of the solar-powered irrigation system is shown in Figure 3. It is assumed that parabolic trough N-S tracking is used for solar collection and water is the working fluid. The aim in the preliminary system design is then to determine:

- maximum operating press., Rankine Cycle, $X_{\mathrm{A}}$ (MPA)

- maximum operating temp., Rankine Cycle, $X_{B}\left({ }^{\circ} \mathrm{K}\right)$

- maximum temp. drop in the solar collectors, $X_{C}\left({ }^{\circ} \mathrm{K}\right)$

- working fluid flow rate, $X_{\mathrm{D}}(\mathrm{kg} / \mathrm{s})$

The design must meet the specifications:

- pumped load (power output) must be as close as possible to $20 \mathrm{~kW}$

- overall efficiency must be maximized as close as possible to $20 \%$

- economic benefits must be maximimzed as near as possible to $\$ 150,000$.

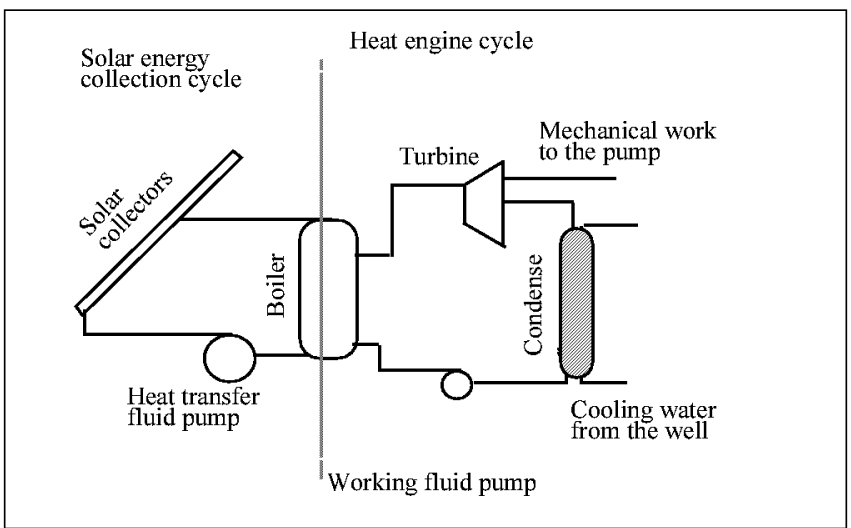

FIGURE 3. SYSTEM CONFIGURATION

The design also must satisfy thermal system requirements, i.e., the maximum temperature in the cycle has to be smaller than the stagnation temperature at all times (The stagnation temperature cannot be less than the maximum temperature corresponding to the maximum pressure). In this work, we combine thermodynamic property prediction software (Shamsundar, 1989) with an economic analysis routine (Bascaran, 1990) for our computer simulation module. Simulation inputs include definitions for components, system parameters involved in system synthesis and the operating environment. System performances, e.g., cycle efficiency, power output, total efficiency and economic benefits are generated. An important aspect of thermal system design is to reduce the variance in system performance caused by the deviation of an uncontrollable operating environment (noise factors). In our case, the noise factors are ambient temperature and level of insolation. Type I robust design can be applied. Another important application of robust design is to reduce the variance of performance caused by deviations in control factors considering that there may be adjustments to these factors in the later stages of design. In other words, it is necessary to find a flat region of design space rather than the optimum. We demonstrate how these two types of robust design can be formulated and solved. 
The first step is to classify the parameters as control factors, noise factors, held-constant factors or responses. The ranges of these parameters and their relationship to the whole solar powered irrigation system are shown in Figure 4. There are four controllable design parameters, representing decisions on thermal cycle design. Two noise factors (uncontrollable parameters) are associated with the unstable operating environment. The heldconstant factors are the constant parameters and the four responses are system performances which can either be considered as goals or constraints. After classifying parameters, it is necessary to fit a surface model for each of the four responses over the space formed by control factors, $\mathrm{X}_{\mathrm{A}}, \mathrm{X}_{\mathrm{B}}, \mathrm{X}_{\mathrm{C}}$, and $\mathrm{X}_{\mathrm{D}}$, and noise factors, $Z_{\mathbf{a}}$ and $Z_{\mathrm{B}}$.

We use the fitted response surface model and a derived function of variance of the response as input to the compromise DSP instead of using an expensive computer simulation at each solution iteration. Using this approach, there is always a tradeoff between the number of experiments used and the accuracy of the estimated model. Studies show that the response-model approach relies on the adequacy of simple models over a small parameter range. A common strategy is to use sequential experimentation, i.e., perform low order screening experiments first over the whole design space to identify appropriate regions of interest, and then build a higher order response model over reduced regions, probably also using a reduced number of factors. Here we assume the reduced parameter ranges are $\mathrm{X}_{\mathrm{A}}$ [2.0-3.0] MPA, $\mathrm{X}_{\mathrm{B}}[450$ $520]^{\circ} \mathrm{K}, X_{\mathrm{C}}[550-650]{ }^{\circ} \mathrm{K}$, and $X_{\mathrm{D}}[0.01-0.05] \mathrm{kg} / \mathrm{s}$. The ranges for the noise factors $Z_{a}$ and $Z_{b}$ are as before.

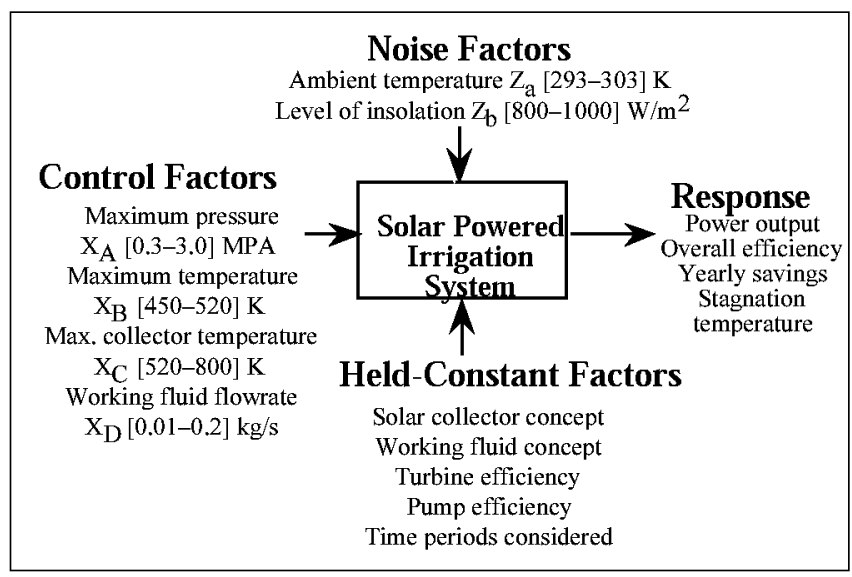

\section{FIGURE 4. CLASSIFICATION OF PARAMETERS}

From low-order screening experiments, we also notice that noise factor $Z_{\mathrm{b}}$ and its associated interactions have no effect on the power and economic benefits while control factor $X_{D}$ and associated interactions do not influence the behavior of efficiency and stagnation temperature. Thus Central Composite Design (CCD) with 43 experiments for 5 factors can be used to construct quadratic response surfaces for each response (Montgomery, 1991). The CCD is composed of a $2^{5}$ (32) factorial design, 10 "star" points and 1 center point. The suggested value for $\alpha$, a parameter varied to meet conditions of equal precision of estimation in all directions, is 2.378. Based on the results of the experiment, the least squares method from regression analysis is used to fit a quadratic surface model:

$$
\begin{aligned}
f\left(x_{1}, \ldots x_{n}\right)=\beta_{0} & +\beta_{1} x_{1}+\ldots+\beta_{n} x_{n}+\gamma_{1} x_{1}^{2}+\ldots+\gamma_{n} x_{n}^{2} \\
& +\beta_{12} x_{1} x_{2}+\ldots+\beta_{n, n-1} x_{n-1} x_{n}
\end{aligned}
$$

A summary of the response surface models for the four system performances is presented in Table 1 . Variables $\mathrm{X}_{\mathrm{A}}, \mathrm{X}_{\mathrm{B}}, \mathrm{X}_{\mathrm{C}}$, $X_{D}, Z_{a}$ and $Z_{b}$ used in the equations are all normalized from -1 to 1 . One benefit of using normalized variables is that the total response variance can be calculated easily when there are several sources of variance. Based on the coefficients of the response model, a designer can see clearly the significance (contributions) of different terms (linear, quadratic and interaction terms). The functions in Table 1 are the reduced models with some trivial effects ignored, e.g., those items with very small coefficients. If, after normalization, the contribution of an item is less than $1 \%$, it is considered trivial. The sequence of items is arranged in descending order of significance.

The normalized quadratic surface model can also help to identify potential control factors for reducing the noise effects by examining the control-noise interactions. For example, to reduce the variance of power caused by the noise factors $\mathrm{Z}_{\mathrm{a}}$, the potential adjustment factors are $\mathrm{X}_{\mathrm{A}}, \mathrm{X}_{\mathrm{B}}$ and $\mathrm{X}_{\mathrm{D}}$. Further, using $X_{D}$ is most effective because the coefficient of $X_{D} Z_{a}$ is greater. Based on the response models, we can formulate compromise DSPs for Type I and Type II robust design applications.

\begin{tabular}{|c|c|}
\hline & Response Model \\
\hline $\begin{array}{c}\text { Power } \\
\text { (objective) } \\
\mathrm{kW}\end{array}$ & 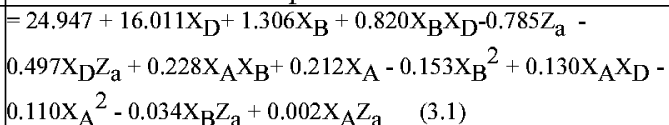 \\
\hline $\begin{array}{l}\text { Efficiency } \\
\text { (objective) }\end{array}$ & $\begin{array}{l}=0.18507+0.01041 \mathrm{X}_{\mathrm{A}}+0.00380 \mathrm{Z}_{\mathrm{b}^{-}}-0.00366 \mathrm{Z}_{\mathrm{a}}-0.00350 \mathrm{X}_{\mathrm{C}^{-}} \\
0.00157 \mathrm{X}_{\mathrm{B}}\end{array}$ \\
\hline $\begin{array}{c}\text { Savings } \\
\text { (objective) } \\
\$\end{array}$ & $\begin{array}{l}=174695.73+112114.69 \mathrm{X}_{\mathrm{D}^{+}}+9133.80 \mathrm{X}_{\mathrm{B}^{+}}+5733.05 \mathrm{X}_{\mathrm{B}} \mathrm{X}_{\mathrm{D}^{-}} \\
5487.76 \mathrm{Z}_{\mathrm{a}}-3478.84 \mathrm{X}_{\mathrm{D}} \mathrm{Z}_{\mathrm{a}}+1586.48 \mathrm{X}_{\mathrm{A}} \mathrm{X}_{\mathrm{B}^{+}} 1486.84 \mathrm{X}_{\mathrm{A}^{-}} \\
1067.42 \mathrm{X}_{\mathrm{B}}{ }^{2}+916.26 \mathrm{X}_{\mathrm{A}} \mathrm{X}_{\mathrm{D}}-768.90 \mathrm{X}_{\mathrm{A}}^{2}-242.88 \mathrm{X}_{\mathrm{B}^{2}} \mathrm{Z}_{\mathrm{a}}{ }^{+} \\
152.40 \mathrm{X}_{\mathrm{A}} \mathrm{Z}_{\mathrm{a}}\end{array}$ \\
\hline $\begin{array}{c}\text { Tstag K } \\
\text { (constraint) }\end{array}$ & $=1873-5 Z_{a}-175 Z_{b}$ \\
\hline
\end{tabular}

\section{TABLE 1. RESPONSE MODELS FOR PERFORMANCE}

Compromise DSP Type I Reducing the variance of system performance caused by the deviation of uncontrollable operating environment is associated with the Type $\mathbf{I}$ robust design application, Table 2. Using the compromise DSP, the problem becomes how to choose $X_{A}, X_{B}, X_{C}$ and $X_{D}$ to reduce the influence from $Z_{\mathrm{a}}$ and $Z_{\mathrm{b}}$ while keeping the performance as close as possible to the targets.

To handle multiple objectives simultaneously, each objective is modeled as a goal. Tradeoffs are implemented by minimizing the total deviation function. As we choose to address separately the issues of bringing the mean to target and minimizing the deviation, for three system performance targets, there will be six goals. $f_{1}-f_{6}$ in the deviation function represent different priority levels, assigned to the six goals. Initially, all goals are at the same priority level and assigned equal weights. The deviation function 
becomes:

$$
\begin{gathered}
\mathrm{Z}=0.167\left(\mathrm{~d}_{1}{ }^{-}+\mathrm{d}_{1}{ }^{+}\right)+0.167\left(\mathrm{~d}_{2}{ }^{-}+\mathrm{d}_{2}{ }^{+}\right)+0.167\left(\mathrm{~d}_{3}{ }^{-}\right)+ \\
0.167\left(\mathrm{~d}_{4}{ }^{-}+\mathrm{d}_{4}{ }^{+}\right)+0.167\left(\mathrm{~d}_{5}{ }^{-}\right)+0.167\left(\mathrm{~d}_{6}{ }^{-}+\mathrm{d}_{6}{ }^{+}\right)
\end{gathered}
$$

Further information about the formulation and solution of compromise DSPs is available in Mistree, et al, $1993 \mathrm{~b}$.

\section{TABLE 2. THE COMPROMISE DSP TYPE I}

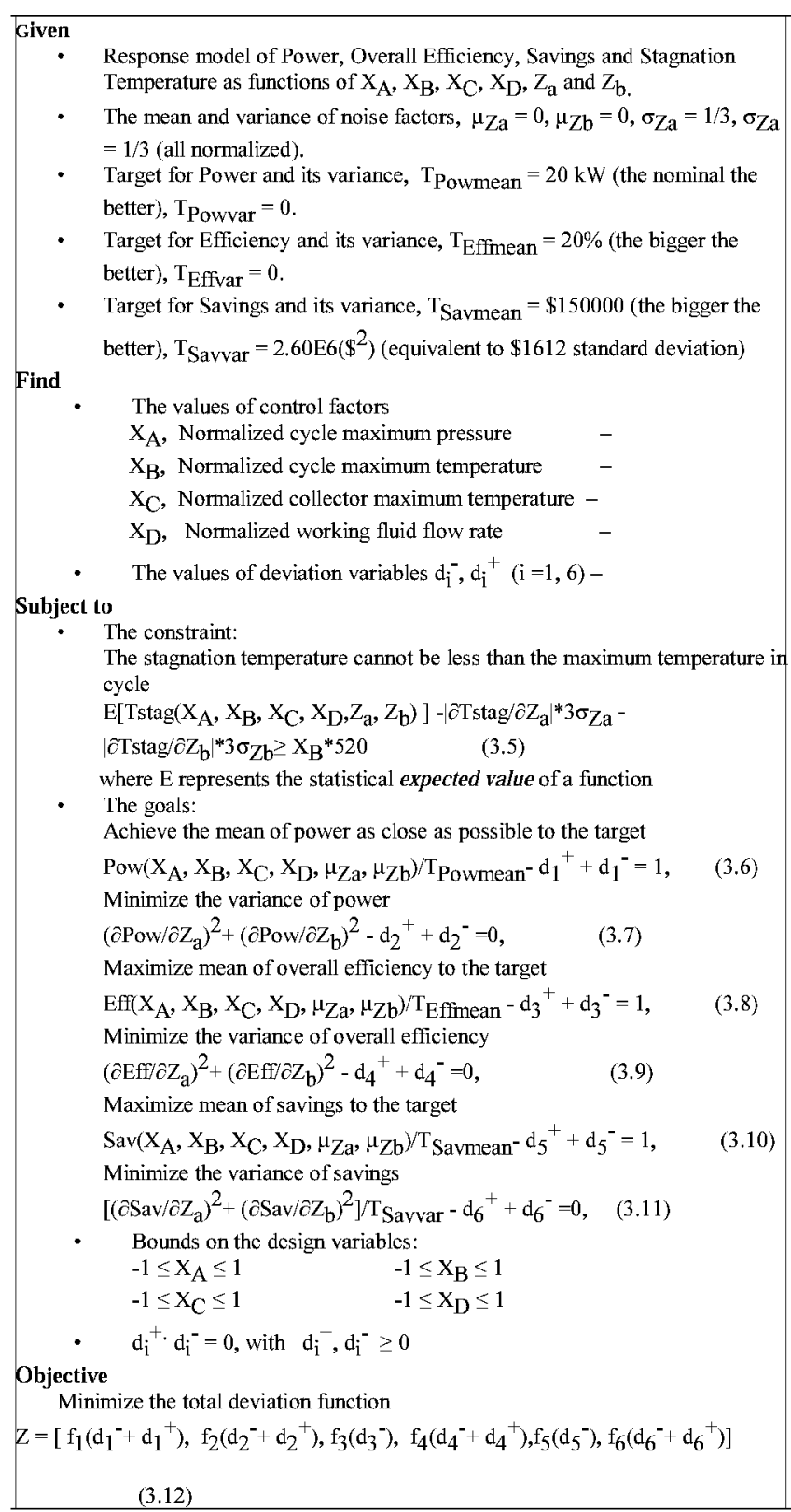

Eqns. (3.5-3.11) can be expanded using the response surface models in Table 1, e.g., using Eqn. (3.1), Eqn. (3.6) becomes:

$\left(24.947+16.011 * \mathrm{X}_{\mathrm{D}}+1.306 * \mathrm{X}_{\mathrm{B}}+0.820 * \mathrm{X}_{\mathrm{B}}{ }^{*} \mathrm{X}_{\mathrm{D}}{ }^{+}\right.$ $0.228 * \mathrm{X}_{\mathrm{A}} * \mathrm{X}_{\mathrm{B}}+0.212 * \mathrm{X}_{\mathrm{A}}-0.153 * \mathrm{X}_{\mathrm{B}}^{2}+0.130 * \mathrm{X}_{\mathrm{A}} * \mathrm{X}_{\mathrm{D}}-$

$\left.0.110^{*} \mathrm{X}_{\mathrm{A}}\right) / \mathrm{T}_{\text {Powmean }}-\mathrm{d}_{1}{ }^{+}+\mathrm{d}_{1}^{-}=1$
Similarly Eqn. (3.7) can be expanded:

$\left(-0.785-0.497 * X_{D^{-}} 0.034 * X_{B}+0.002 * X_{A}\right)^{2}-d_{2}{ }^{+}+d_{2}{ }^{-}=0$

In the compromise DSP, to calculate the performance variance, instead of using ${ }_{i=1}^{k}\left(\frac{\partial f}{\partial z_{i}}\right)^{2} \sigma_{z_{i}}^{2}$, only ${ }_{i=1}^{k}\left(\frac{\partial f}{\partial z_{i}}\right)^{2}$ is required. When there are several noise factors, $z_{i}$, after normalization, $\sigma_{\mathrm{zi}}$ has the same value if the standard deviation is proportional to the range of design variables. This is equal to $1 / 3$ assuming that the deviation follows a normal distribution. With respect to the goal of minimizing variance, it is desirable that the target approach zero. However, it is not always advisable to use zero as a target because tremendous resources are often required to reduce variance to zero. In this work, we first calculate a reasonable minimum value for the variance of a response without considering any other goals and use this value as the target for the variance goal. For Power and Efficiency zero becomes the target. The target for variance of economic benefits is $2.60 \mathrm{E}+6\left(\$^{2}\right)$.

The compromise DSP in Table 2 is solved using the DSIDES software (Mistree, et al., 1993a) and the results are:

$\mathrm{X}_{\mathrm{A}}=3.0 \mathrm{MPA}, \mathrm{X}_{\mathrm{B}}=450 \mathrm{~K}, \mathrm{X}_{\mathrm{C}}=550 \mathrm{~K}$ and $\mathrm{X}_{\mathrm{D}}=0.0258(\mathrm{~kg} / \mathrm{s}) \mathrm{At}$ this solution point, the values of mean and variance of each performance with the total deviation function value, $Z$, equal to 0.02577 :

$$
\begin{array}{ll}
\text { Pow }_{\text {mean }}=20.0027(\mathrm{~kW}) \text { Pow }_{\text {var }}= & 0.0068\left(\mathrm{~kW}^{2}\right) \\
\mathrm{Eff}_{\text {mean }}=19.45 \% & \mathrm{Eff}_{\mathrm{var}}=4.93 \mathrm{E}-06 \\
\mathrm{Sav}_{\text {mean }}=141143(\$) & \mathrm{Sav}_{\mathrm{var}}=3.3 \mathrm{E}+06\left(\$^{2}\right)
\end{array}
$$

To confirm the results, we compare values from the response model with those from a complete simulation, Table 3.

\section{TABLE 3. CONFIRMATION OF RESULTS}

\begin{tabular}{|l|c|c|c|}
\hline & $\begin{array}{c}\text { Values from the second- } \\
\text { order response model }\end{array}$ & $\begin{array}{c}\text { Values from original } \\
\text { simulation }\end{array}$ & Error \\
\hline Mean Power $(\mathrm{kW})$ & 20.003 & 19.680 & $1.6 \%$ \\
\hline Mean Efficiency & $19.45 \%$ & $19.45 \%$ & 0 \\
\hline Mean Savings (\$) & 141143 & 137795 & $2.4 \%$ \\
\hline
\end{tabular}

TABLE 4. GOAL PRIORITIES FOR MEAN AND VARIANCE

\begin{tabular}{|c|c|c|c|}
\hline & Scenario I & Scenario II & Scenario III \\
\hline $\begin{array}{c}\text { Preemptive formulation of deviationevel 1-all goals } \\
\text { function }\end{array}$ & $\begin{array}{c}\text { Level 1-Mean } \\
\text { Level 2- } \\
\text { Variance }\end{array}$ & $\begin{array}{c}\text { Level 1- } \\
\text { Variance } \\
\text { Level 2-Mean }\end{array}$ \\
\hline
\end{tabular}




\begin{tabular}{|l|c|c|c|}
\hline System variables & & & \\
$\mathrm{X}_{\mathrm{A}}(\mathrm{MPA})$ & 3.0 & 3.0 & 3.0 \\
$\mathrm{X}_{\mathrm{B}}(\mathrm{K})$ & 450 & 450 & 450 \\
$\mathrm{X}_{\mathrm{C}}(\mathrm{K})$ & 550 & 550 & 550 \\
$\mathrm{X}_{\mathrm{D}}(\mathrm{kg} / \mathrm{s})$ & 0.0258 & 0.0258 & 0.01 \\
Mean on Target & & & \\
$\quad$ Power (Target $20 \mathrm{~kW})$ & 20.0027 & 20.0027 & 8.04411 \\
$\quad$ Efficiency Target 20\%) & $19.45 \%$ & $19.45 \%$ & $19.45 \%$ \\
$\quad 141143$ & 141143 & 57397.9 \\
Savings (Target $\$ 150,000)$ & & & \\
"Reducing Variance" & 0.0068 & 0.0068 & $9.405 \mathrm{E}-03$ \\
$\quad$ Power (Target 0) & $4.93 \mathrm{E}-06$ & $4.93 \mathrm{E}-06$ & $4.927 \mathrm{E}-06$ \\
$\quad$ Eff. (Target 0) & $3.3 \mathrm{E}+06$ & $3.3 \mathrm{E}+06$ & 460465 \\
Savings (Target 2.6E +06$)$ & & & \\
Dev. Function Value, $Z$ & 0.02577 & 0.0286434 & 0.0031053 \\
$\quad$ level 1 & & 0.0222924 & 0.410113 \\
$\quad$ level 2 & \multicolumn{2}{|c}{} \\
\hline \multicolumn{2}{|c}{}
\end{tabular}

In addition to the comparison at solution points, we also use other random points to check the accuracy of our predictor. This shows that the accuracy of our response model is satisfactory within the range of study. This increases our confidence in using the response model as a fast analysis module for further study.

When considering multiple aspects of quality, designers may have different preferences for whether it is more important to bring the mean on target or reduce variation. In the compromise DSP, different design scenarios can be modeled by assigning goals different priority levels. In Table 4 , the results of three different preemptive formulations of deviation function are given.

It is interesting that the result for Scenario II are the same as those for Scenario I, Table 4. The means obtained are very close to their targets in Scenario I, so there is not much room for improvement in Scenario II. In Scenario III, variation has been reduced significantly, however, this is achieved by sacrificing the goals of bringing the mean on target. Studies on different goal priority levels for mean and variance can help designers obtain a clearer idea of what is possible and understand the major obstacles to achieving a robust design.

Compromise DSP Formulation II Given that $\mathrm{X}_{\mathrm{A}}, \mathrm{X}_{\mathrm{B}}, \mathrm{X}_{\mathrm{C}}$ and $\mathrm{X}_{\mathrm{D}}$ are system-level design variables, adjustments are possible after the design at the component level is finalized. Type II robust design reduces the variance of performance due to deviations of control factors. Suppose that within the design parameter range, e.g., $\mathrm{X}_{\mathrm{A}}[2.0-3.0 \mathrm{MPA}], \mathrm{X}_{\mathrm{B}}\left[450-520^{\circ} \mathrm{K}\right], \mathrm{X}_{\mathrm{C}}$ $\left[550-650^{\circ} \mathrm{K}\right]$, and $\mathrm{X}_{\mathrm{D}}[0.01-0.05 \mathrm{~kg} / \mathrm{s}]$, the possible deviation for each variable is constant, after normalization, say $\Delta \mathrm{X}_{\mathrm{A}}=\Delta \mathrm{X}_{\mathrm{B}}=\Delta \mathrm{X}_{\mathrm{C}}=\Delta \mathrm{X}_{\mathrm{D}}=0.2$, then the compromise DSP for robust design in this new situation is given in Table 5 . We assume that noise factors $Z_{a}$ and $Z_{b}$ become constants at their means $Z_{a}=298 K$ and $Z_{b}=900 \mathrm{~W} / \mathrm{m}^{2}$.

The target for each variance goal is the best possible value obtained without considering the other goals. We use three different deviation functions to test solutions for different scenarios, Table 6. In Scenario I, all the goals are at the same priority level with equal weights; while in Scenario II, six goals are on the same priority level with higher weights for reducing the variance. In Scenario III all six goals have the same priority but higher weights are assigned to bring the mean on target.

Based on the results from Table 6, we observe:

- The results for $X_{B}, X_{C}$, and $X_{D}$ are stable under different scenarios. However, $X_{A}$ jumps between its lower and upper bounds. Thus a higher cycle pressure is preferred for bringing the mean on target, and a lower cycle pressure is preferred for reducing variance.

- For Power, the goal of bringing the mean on target can always be achieved exactly, while for Efficiency, the mean on target goal must be sacrificed if reducing the variance is assigned a higher weight. For Saving, it is always difficult to bring the mean on target.

- The goals associated with reducing variance can be better achieved when they are given a higher weight. The variance in efficiency is always a small constant because it is not a function of design variables.

These observations match the mathematical structure of the response model, e.g., for the second observation, the equation of efficiency indicates that the larger $\mathrm{X}_{\mathrm{A}}$, the easier it is to bring it on target. However, this contradicts the notion of reducing variance of the other responses. If variance reduction is assigned a higher weight, an appropriate trade-off results.

\section{TABLE 5. THE COMPROMISE DSP TYPE II}

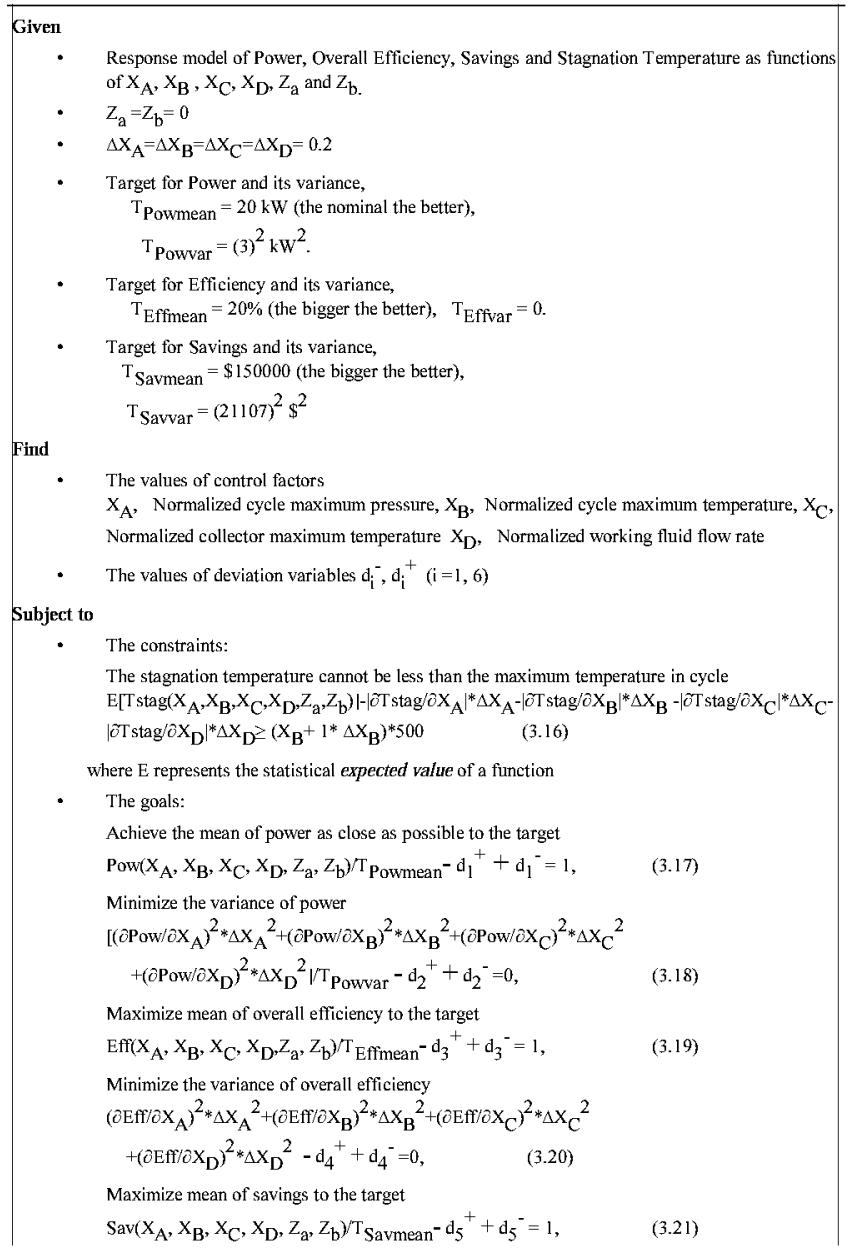




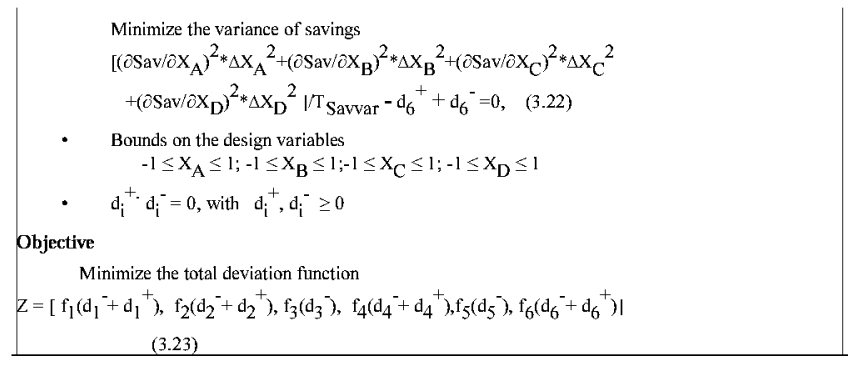

\section{CLOSURE}

A comprehensive and rigorous procedure is introduced to overcome some of the limitations of existing experiment-based and nonlinear programming approaches to robust design. We incorporate useful ideas from the existing approaches and to handle two different types of robust design. The integration of Response Surface Methodology with the compromise DSP appears to have several advantages:

- Engineering constraints can be introduced and there is great flexibility for studying tradeoffs among multiple design objectives.

- The interaction effects and nonlinear effects can be considered using the response surface model, e.g., quadratic surfaces. Compared to Taguchi's linear model approach, this approach yields more accurate results.

- Response surface models can serve as fast analysis modules for different types of robust design application. By introducing a parameter as a variable in the response surface model, we extend our scope of study.

- The compromise DSP addresses individually the issues of bringing the mean on target and reducing variation. This provides designers more flexibility to make decisions based on different robust design criteria.

Robust design procedures can be applied to various kinds of engineering problems. We demonstrate the application of this method to the design of a thermal system. In the future, we will apply quality concepts to improving efficiency and effectiveness in designing more complex systems. Potential applications include decomposition of complex system design into subproblems using Type I robust design, and the generation of flexible design specifications for increasing design freedom using the Type II robust design. We are in the process of developing a Robust Concept Exploration Model, RCEM, which is used to support the rapid evaluation of different design alternatives to generate competitive top-level design specifications. Improving the response surface model by narrowing the design space to the region of greatest interest is an important part of this procedure.

\section{TABLE 6. RESULTS FOR TYPE ॥}

\begin{tabular}{|l|c|c|c|}
\hline System variables & & & \\
$\mathrm{X}_{\mathrm{A}}(\mathrm{MPA})$ & & & \\
$\mathrm{X}_{\mathrm{B}}(\mathrm{K})$ & 2.974 & 2.000 & 2.973 \\
$\mathrm{X}_{\mathrm{C}}(\mathrm{K})$ & 450 & 450 & 450 \\
$\mathrm{X}_{\mathrm{D}}(\mathrm{kg} / \mathrm{s})$ & 550 & 550 & 550 \\
Mean & 0.0256 & 0.0255 & 0.0256 \\
Power & 20.0 & 20.0 & 20.0 \\
Efficiency & $20 \%$ & $17.97 \%$ & $20 \%$ \\
Savings & 140085 & 140062 & 140085 \\
Variance & & & \\
Power & 9.492 & 9.132 & 9.492 \\
Efficiency & $4.92 \mathrm{E}-06$ & $4.92 \mathrm{E}-06$ & $4.92 \mathrm{E}-06$ \\
$\quad$ Savings & $4.654 \mathrm{E}+08$ & $4.477 \mathrm{E}+08$ & $4.654 \mathrm{E}+08$ \\
Deviation Function & 0.0276 & 0.0213 & 0.0253 \\
Value Z & & & \\
\end{tabular}

Preemptive formulations of the deviation function in Table 6:

Scenario I: $\mathrm{Z}=0.167\left(\mathrm{~d}_{1}^{-}+\mathrm{d}_{1}{ }^{+}\right)+0.167\left(\mathrm{~d}_{2}^{-}+\mathrm{d}_{2}{ }^{+}\right)+0.167\left(\mathrm{~d}_{3}{ }^{-}\right)$

$$
+0.167\left(\mathrm{~d}_{4}{ }^{-}+\mathrm{d}_{4}{ }^{+}\right)+0.167\left(\mathrm{~d}_{5}{ }^{-}\right)+0.167\left(\mathrm{~d}_{6}{ }^{-}+\mathrm{d}_{6}{ }^{+}\right)
$$

Scenario II: $Z=0.1\left(\mathrm{~d}_{1}^{-}+\mathrm{d}_{1}{ }^{+}\right)+0.233\left(\mathrm{~d}_{2}^{-}+\mathrm{d}_{2}{ }^{+}\right)+0.1\left(\mathrm{~d}_{3}{ }^{-}\right)$

$$
+0.233\left(\mathrm{~d}_{4}{ }^{-}+\mathrm{d}_{4}{ }^{+}\right)+0.1\left(\mathrm{~d}_{5}{ }^{-}\right)+0.233\left(\mathrm{~d}_{6}{ }^{-}+\mathrm{d}_{6}{ }^{+}\right)
$$

Scenario III: $\mathrm{Z}=0.233\left(\mathrm{~d}_{1}^{-}+\mathrm{d}_{1}{ }^{+}\right)+0.1\left(\mathrm{~d}_{2}^{-}+\mathrm{d}_{2}{ }^{+}\right)+0.233\left(\mathrm{~d}_{3}^{-}\right)$

$$
+0.1\left(\mathrm{~d}_{4}{ }^{-}+\mathrm{d}_{4}{ }^{+}\right)+0.233\left(\mathrm{~d}_{5}{ }^{-}\right)+0.1\left(\mathrm{~d}_{6}{ }^{-}+\mathrm{d}_{6}{ }^{+}\right)
$$

\section{ACKNOWLEDGMENTS}

We are grateful to Professor N. Shamsunder, University of Houston, for providing the thermodyanmic software. Wei Chen is supported by The Woodruff School of Mechanical Engineering, Georgia Tech. We gratefully acknowledge NSF grant DDM-9396503 and NASA Grant NAG-1-1564.

\section{REFERENCES}

Bascaran, E., 1990, "A Conceptual Model for the Design of Thermal Systems: Concurrent Decisions in Designing for Concept," Ph.D. Dissertation, Department of Mechanical Engineering, University of Houston, Houston, Texas.

Box, G., 1988, "Signal-to-Noise Ratios, Performance Criteria, and Transformations," Technometrics, Vol 30, No 1, pp. 1-18.

Chen, W., Allen, J.K. and Mistree, F., 1994, "Robust Concurrent Concept Selection and System Synthesis," Advances in Design Automation, ASME DE-Vol. 69-1, pp. 141-149.

Ignizio, J.P., 1985, "Multiobjective Mathematical Programming via the MULTIPLEX Model and Algorithm," Eur. Journal of Operational Research, Vol. 22, pp. 338-346.

Khuri, A. and Cornell, J. A., 1987, Response Surfaces: Designs and Analysis, Marcel Dekker Inc., New York.

Mistree, F., Hughes, O.F. and Bras, B.A., 1993a, "The Compromise Decision Support Problem and the Adaptive Linear Programming Algorithm," Structural Optimization: Status and Promise, AIAA, Washington, D.C., pp. 247-286.

Mistree, F., Lautenschlager, U. and Erikstad, S.O., 1993b, "Simulation Reduction using the Taguchi Method", NASA Contractor Report, CR 93-4542.

Montgomery, D.C., 1991, Design and Analysis of Experiments, John Wiley, New York.

Nair, V.N., 1992, "Taguchi's Parameter Design: A Panel Discussion," Technometrics, Vol. 34, No. 2, pp. 127-161.

Otto, K.N. and Antonsson, E.K., 1991, "Extensions to the Taguchi Method of Product Design," Third International Conference on Design Theory and Methodology, (Stauffer, L. A., 
Ed.), Miami, Florida, pp. 21-30.

Phadke, M.S., 1989, Quality Engineering using Robust

Design, Prentice Hall, Englewood Cliffs, New Jersey.

Ramakrishnan, B. and Rao, S.S., 1991, "A Robust Optimization Approach Using Taguchi's Loss Function for Solving Nonlinear Optimization Problems," Advances in Design Automation, ASME DE-32-1, pp. 241-248.

Shamsundar, N., 1989, "University of Houston Thermodynamic Properties Software," Department of Mechanical Engineering, University of Houston.

Shoemaker, A.C., Tsui, K.-L. and Wu, J., 1991, "Economical Experimentation Methods for Robust Design," Technometrics, Vol. 33, No. 4, pp. 415-427.

Sundaresan, S., Ishii, K. and Houser, D.R., 1993, "A Robust Optimization Procedure with Variations on Design Variables and Constraints," Advances in Design Automation, ASME DE-Vol. 69-1, pp. 379-386.

Tsui, K.-L., 1992, "An Overview of Taguchi Method and Newly Developed Statistical Methods for Robust Design," IIE Transactions, Vol. 24, No. 5, pp. 44-57.

Welch, W.J., Yu, T.K., Kang, S.M. and Wu, J., 1990, "Computer Experiments for Quality Control by Parameter Design," Quality Technology, Vol. 22, pp. 15-22. 